\title{
Cosmetic and Reconstructive Breast Surgery in Adolescents: Psychological, Ethical, and Legal Considerations
}

\author{
Canice E. Crerand, $\mathrm{PhD}^{1,2} \quad$ Leanne Magee, $\mathrm{PhD}^{1}$ \\ ${ }^{1}$ Division of Plastic and Reconstructive Surgery, The Children's \\ Hospital of Philadelphia, Philadelphia, Pennsylvania \\ 2 Division of Plastic Surgery, Department of Surgery, The Edwin and \\ Fannie Gray Hall Center for Human Appearance, Perelman School of \\ Medicine, University of Pennsylvania, Philadelphia, Pennsylvania
}

\author{
Address for correspondence Canice E. Crerand, PhD, Division of \\ Plastic and Reconstructive Surgery, The Children's Hospital of \\ Philadelphia, Wood Ambulatory Care Building, First Floor, 34th and \\ Civic Center Blvd., Philadelphia, PA 19104 \\ (e-mail: crerand@email.chop.edu).
}

Semin Plast Surg 2013;27:72-78.

\begin{abstract}
Keywords

- plastic surgery

- breast

- adolescence

- psychosocial outcomes

- ethics

Cosmetic and reconstructive breast surgery has increased in popularity among adolescents in the United States. As more adolescents pursue these procedures, an understanding of the psychological aspects of these surgeries becomes increasingly important for the benefit of both patients and providers. The authors review the psychological aspects of cosmetic and reconstructive surgical breast procedures as they pertain to adolescents, including augmentation mammoplasty, gynecomastia correction, breast reduction, and asymmetry correction. They include a discussion of the medicolegal and ethical implications of these procedures and recommendations for clinical management.
\end{abstract}

According to the American Society of Plastic Surgeons (ASPS), more than 76,000 cosmetic surgical procedures were performed on teens ages 13 to 19 in 2011, accounting for $5 \%$ of all cosmetic surgical procedures performed in the United States that year. ${ }^{1}$ Breast augmentation and breast reduction (in adolescent boys) were among the top five procedures performed on teens. ${ }^{1}$ Surgeons and mental health professionals have long been interested in the psychological characteristics and the psychosocial outcomes of adults who undergo plastic surgery, which has led to a relatively well-developed body of literature. ${ }^{2,3}$ In contrast, there has been very little research on the psychological characteristics of adolescents who seek plastic surgery, and, similarly little thoughtful consideration of the appropriateness of performing these procedures on individuals whose bodies and body images are still developing. ${ }^{4}$

Body image, defined as perceptions and attitudes toward one's own physical appearance, is the largest contributor to self-esteem and self-concept among children and adolescents. ${ }^{5}$ Adolescence is a key time in body-image development because of the normative developmental challenges that influence and are influenced by body image, such as pubertal development, identity formation, and emergence of sexuality. ${ }^{5}$ Body image may motivate many self-improvement behaviors, including dieting, exercise, and plastic surgery. ${ }^{2,3}$ Among adult cosmetic surgery patients, studies have documented increased body image dissatisfaction prior to surgery ${ }^{6,7}$ and improvements in body image within the first two postoperative years. ${ }^{8,9}$

Far less is known about body image concerns among adolescent plastic surgery patients. In one of the few studies of adolescent plastic surgery patients, adolescents reported increased body-image dissatisfaction with the feature for which they desired treatment. ${ }^{10}$ The majority of adolescents were judged to be psychologically healthy, and they reported postoperative satisfaction with their appearance and improved self-confidence. ${ }^{11}$ Thus, there is preliminary evidence to suggest that adolescent patients are similar to adults in that the majority appear to be psychologically appropriate for surgery and may experience psychological benefit postoperatively. Nonetheless, plastic surgery may not be appropriate for all adolescents who request it, and there are some important developmental, legal, and ethical issues related to adolescent plastic surgery populations that require careful consideration.
Issue Theme The Adolescent Breast; Guest Editors, Valerie Lemaine, MD, MPH, FRCSC and Patricia S. Simmons, MD
Copyright @ 2013 by Thieme Medical Publishers, Inc., 333 Seventh Avenue, New York, NY 10001, USA. Tel: +1(212) 584-4662.
DOI http://dx.doi.org/ 10.1055/s-0033-1343999. ISSN $1535-2188$. 
Here we review the psychological aspects of cosmetic and reconstructive surgical breast procedures, including augmentation mammoplasty, gynecomastia correction, breast reduction, and asymmetry correction, as they pertain to adolescents. We also examine the legal and ethical considerations related to plastic surgery and adolescents. We conclude with strategies for clinical management.

\section{Psychological Aspects of Cosmetic and Reconstructive Procedures}

\section{Cosmetic Procedures}

\section{Breast Augmentation}

Approximately 8,000 young women ages 13 to 19 underwent breast augmentation in 2011, accounting for 3\% of the 307,000 women who had the procedure in the same year. ${ }^{1}$ The enduring popularity of breast augmentation surprises many individuals, particularly given that the U.S. Food and Drug Administration (FDA) has not approved breast implants for use in adolescents under the age of 18 due to concerns that they may not yet have finished physical development or have the emotional maturity to manage the physical and psychological outcomes of surgery. ${ }^{12}$ For similar reasons, the ASPS does not endorse the use of breast implants for cosmetic purposes in adolescents under the age of $18 .^{13}$ Despite these recommendations, it is still possible for surgeons to perform cosmetic breast augmentation on patients under 18 years of age with parental consent as an off-label use of the device. Breast implants may also be used to correct congenital or acquired breast deformities (see Asymmetry Correction section below).

There is limited empirical research investigating the psychological characteristics of young women who undergo breast augmentation, likely related to the controversy surrounding the use of breast implants in adolescents. In contrast, a large body of research has examined the psychosocial characteristics and postoperative outcomes of adult women who receive cosmetic breast implants. ${ }^{14,15}$ Although a full review of this literature is beyond the scope of this article, there are some findings that may have relevance for adolescents who pursue breast augmentation.

Preoperatively, adult women who request breast augmentation typically report heightened dissatisfaction with their breasts. ${ }^{16}$ Compared with women who do not have breast implants, women with breast implants are more likely to have had more overall lifetime sexual partners, report a greater use of oral contraceptives, be younger at their first pregnancy, and have a history of terminated pregnancies as compared with other women. ${ }^{14,15}$ They also have been found to be more frequent users of alcohol and tobacco, as well as to have a higher divorce rate. ${ }^{14,15}$ Finally, they have been reported to have a below-average body weight, leading to concern that some may be experiencing eating disorders. ${ }^{14-16}$

Postoperatively, the vast majority of women appear to be satisfied with the outcome of breast augmentation, ${ }^{8,17}$ and there is some evidence for improvements in self-esteem and depressive symptoms. ${ }^{17}$ Women who undergo breast aug- mentation also report improvements in their body image postoperatively. ${ }^{8,17}$ Body-image improvements, however, may be tempered by the occurrence of a postoperative complication, which occurs in 24 to $31 \%$ of patients. ${ }^{8,18}$

Most notably, several studies have found that the suicide rate among adult breast augmentation patients is two to three times higher compared with either patients who underwent other cosmetic surgical procedures or population estimates, though the nature of this relationship is unclear. ${ }^{19}$ One study found that women who received breast implants had an increased prevalence of preoperative psychiatric hospitalizations compared with women who underwent other forms of plastic surgery, suggesting that the increase suicide rate may reflect some underlying psychopathology rather than a direct relationship with the implants. ${ }^{20}$ However, not all of the studies were able to determine whether mental health issues developed before or after surgery. ${ }^{19}$ Though equivocal, these findings highlight the need for young women and adolescents seeking cosmetic breast augmentation to be screened for depression and substance use, as well as disorders with body-image components, such as eating disorders and body dysmorphic disorder. Adolescents with a history of psychiatric hospitalizations or psychopathology should have a mental health evaluation prior to breast augmentation surgery. ${ }^{19}$

\section{Reconstructive Procedures}

\section{Gynecomastia Correction}

In 2011, over 14,000 breast reduction procedures were performed in the United States on adolescent boys for the correction of gynecomastia. ${ }^{1}$ Physiological (e.g., imbalance of estrogen and androgen) and pathological (e.g., endocrine disorders, tumors, substance abuse) etiologies of gynecomastia have been identified. ${ }^{21}$ Surgical correction is aimed at male chest contour reconstruction, histological clarification of suspicious breast lesions, and reduction of pain and discomfort associated with hypertrophic breast tissue. ${ }^{22}$ Surgical correction can result in significant functional and aesthetic improvements for affected individuals.

Emotional distress and shame about appearance are considered to be indications for surgery. ${ }^{22,23}$ Adolescent boys with this condition frequently experience significant psychosocial problems because of the gender-incongruent appearance of their chests, including low self-esteem, body-image disturbances, and teasing related to breast enlargement. ${ }^{21,23,24}$ Many affected adolescent boys camouflage the appearance of their chests by wearing loose or oversized shirts or binding their chests with tape or other restrictive materials. Avoidance of situations and activities in which their chest may be exposed (e.g., locker room, athletic activities) is also common. Case reports have described social isolation, eating pathology, depressive symptoms, and social anxiety among affected adolescent boys. ${ }^{25}$ A recent retrospective study of 24 adolescent boys ages 10 to 18 seeking surgery for gynecomastia correction found that $100 \%$ of patients met diagnostic criteria for a formal psychiatric condition, primarily adjustment disorder, but also anxiety 
disorders and dysthymia, and reported significantly higher levels of anxiety, depression, and social phobia compared with the general population. ${ }^{26}$

Though many of the psychosocial problems associated with gynecomastia are thought to resolve postoperatively, empirical evaluation of postoperative psychosocial outcomes is very limited. Clinical experience suggests that surgery does not improve psychosocial functioning for all. Surgery results in some degree of scarring that can be problematic for some patients. Furthermore, some patients report dissatisfaction with their postoperative outcome even after technically successful procedures. ${ }^{23,24}$ Such reports suggest that psychosocial factors such as body image may play an important role in predicting postoperative outcomes. Another underinvestigated issue is substance use and abuse. Marijuana, anabolic steroids, and other drugs can cause gynecomastia, ${ }^{21}$ and substance use can be a marker for psychiatric problems. More research is clearly needed to examine the pre- and postoperative psychosocial functioning of adolescent boys with gynecomastia.

\section{Female Breast Reduction Surgery}

In 2010, 4,645 adolescent girls underwent breast reduction surgery, also known as reduction mammaplasty, to treat macromastia or juvenile breast hypertrophy. ${ }^{27}$ Most candidates desire to have breasts of average size that are proportional to the rest of their bodies. Young women seeking breast reduction commonly report pain and discomfort associated with the size of their breasts as a primary motivation for surgery, including significant back and neck pain, headaches due to neck strain, grooving in their shoulders from bra straps, rashes and skin breakdown, poor posture, and in extreme cases, scoliosis. ${ }^{28,29}$ These symptoms can contribute to interference with participation in sports and exercise. In addition, many young women with macromastia report difficulty finding bras, swimsuits, tops, and dresses that fit and are fashionable among peers.

Significant psychosocial concerns, including body-image dissatisfaction, symptoms of anxiety and depression, low selfesteem, and social isolation may also influence the decision to reduce breast size, particularly among younger women. ${ }^{30}$ Teasing and bullying from peers and unwanted attention, particularly from adolescent boys, can be significant concerns for affected girls. For optimal surgical results, many surgeons delay breast reduction surgery until the late teens, when breast development is completed. In cases where symptoms are severe, surgery may be performed earlier, though subsequent changes in breast size and shape have been reported. ${ }^{31}$

Adolescent girls with macromastia report lower self-esteem and health-related quality of life, greater symptoms of pain and discomfort associated with their breasts, and higher risk of eating-disordered thoughts and behaviors compared with peers without macromastia. ${ }^{32}$ Studies suggest that breast-reduction patients are highly satisfied with their postoperative outcomes, with greater than $90 \%$ reporting that they would have surgery again or recommend it to others. $^{33,34}$ Postoperative improvements in self-esteem, body image, anxiety, depressive symptoms, and quality of life have also been reported. ${ }^{30,35-37}$

Macromastia is often associated with obesity. Greater than $30 \%$ of adults with macromastia are obese. ${ }^{38,39}$ A recent study of adolescents with macromastia found a similar rate of overweight and obesity, but also found that the negative impacts of macromastia on health-related quality of life, selfesteem, and breast symptoms were independent of weight. ${ }^{32}$ In studies of women with macromastia and obesity, weight loss has been shown to be an ineffective nonsurgical tool for reducing symptoms associated with macromastia, making surgery the treatment of choice for women. ${ }^{40}$ Though no similar standards of care currently exist for adolescent women, some studies indicate that much like adult patients, adolescent patients have successful outcomes with reduction mammaplasty, ${ }^{29,41-43}$ and satisfaction with postoperative results may persist into adulthood. ${ }^{42,44}$ Postoperatively, decreased physical symptoms and improvements in body image may allow adolescent girls to more easily engage in exercise and healthy weight maintenance behaviors, though empirical studies have yet to be conducted.

Macromastia has also been associated with eating disorders. ${ }^{45,46}$ In two case series exploring the effects of breast reduction in adolescent and young adult women with bulimia nervosa, patients attributed their eating disordered behaviors to a desire to reduce the size of their breasts and to achieve a more proportionate body. ${ }^{45,46}$ Most patients experienced postoperative improvements in their eating-disorder symptoms, which were evident up to10 years postoperatively. ${ }^{47}$ These findings suggest that large breast size may play a role in the etiology of eating disorders for some women, and breastreduction surgery may play a role in reducing eating pathology. Given that these findings are based on case reports, it is premature to conclude that breast-reduction surgery is an effective treatment for eating pathology. However, mental health professionals should be aware of the risk for eating disorders in this patient population.

\section{Breast Asymmetry Correction}

Hypoplastic breast anomalies can have congenital, developmental, or acquired etiologies and range from slight underdevelopment to complete absence of the breast tissue. ${ }^{21,48,49}$ Typically, breast hypoplasia manifests unilaterally, leading to breast asymmetry. Although most young adolescents will have some breast asymmetry because of familial or genetic factors influencing puberty's timing and breast growth rate, symmetry is eventually achieved in $75 \%$ of women by late adolescence. ${ }^{21}$ However, for adolescents with one or more cup-size disparity, a unilateral augmentation or reduction, or some combination of procedures, may be recommended. Surgical intervention in younger patients is often delayed due to concerns about disrupting subsequent breast development or weight changes in adolescence and early adulthood, which might compromise postoperative cosmetic outcomes. ${ }^{50}$ Reconstruction efforts are generally focused on achieving natural-appearing symmetric breasts ${ }^{48}$ and are often indicated to address psychosocial distress associated with breast hypoplasia. ${ }^{51}$ Unfortunately, statistics are not 
available regarding the number of breast asymmetry-correction procedures performed each year in the United States.

Although anecdotal reports suggest that breast-size discrepancies in adolescents can lead to ridicule, low confidence, social withdrawal, and depression, and thus significantly impact quality of life, ${ }^{52}$ empirical investigation of psychosocial functioning in adolescents undergoing breast reconstruction for asymmetry is exceedingly limited. One retrospective study of 14 women under age 25 who underwent staged reconstruction with tissue expansion to correct severe breast asymmetry (e.g., Poland syndrome) found that most were satisfied with their surgical outcome; however, objective measures of satisfaction or psychosocial outcomes were not used. ${ }^{50}$ There is a clear need for additional empirical research exploring the psychosocial status and body-image concerns among young women undergoing reconstructive surgery for breast hypoplasia.

\section{Legal and Ethical Issues Related to the Clinical Management of the Adolescent Breast Surgery Patient}

Adolescents represent a special plastic surgery patient population because they are still experiencing physical and psychological growth and development. As such, there are unique developmental, ethical, and legal concerns that should be taken into consideration when breast procedures are requested by adolescents. Here, we describe some of the challenges inherent to performing breast surgery in adolescents and detail some of the factors that should be evaluated preoperatively, namely knowledge of the procedure and informed consent, motivations and expectations for surgery, psychological functioning, and body image.

\section{Legal and Ethical Considerations}

Adolescence is a developmental stage characterized by significant physical, psychological, cognitive, and social changes, which result in vulnerabilities to peer influences and body-image fluctuations. ${ }^{5}$ Although adolescents are experiencing emerging autonomy in a variety of areas, they are still typically dependent on their parents or guardians for physical, emotional, and financial support. ${ }^{53}$ Furthermore, there is now a growing body of literature that suggests that adolescents' cognitive abilities, including the ability to consider long-term consequences for one's actions, are still developing. ${ }^{54}$ Adolescence is known to be a time when individuals often engage in risky behavior (e.g., experimentation with drugs and alcohol) and may have a difficult time appreciating long-term consequences of behavior (e.g., engaging in unprotected sexual intercourse may lead to disease or pregnancy). Indeed, studies of brain development indicate that the regions of the brain in the prefrontal cortex that are responsible for inhibiting risky behavior are not fully developed until the early to mid-20s. ${ }^{53,54}$ It is also notable that many psychiatric disorders (e.g., mood and psychotic disorders) often emerge during adolescence. ${ }^{55}$ All of these issues can potentially impact and limit an adolescent's ability to make an informed decision about plastic surgery and accurately appreciate the short- and long-term risks and benefits of these procedures.

Because of these vulnerabilities, adolescents under the age of 18 legally cannot consent for surgery; parents or legal guardians must provide consent, although adolescents between the ages of 12 to 17 years should be able to give assent for the procedure. These concerns also underpin the FDA's restrictions on the use of saline implants for women under 18 and use of silicone implants for women under 22 as well as the recommendation from ASPS that cosmetic breast augmentation should be reserved for individuals 18 years of age and older. ${ }^{12,13}$

From an ethical standpoint, surgeons should be mindful of the principles of autonomy, beneficence, nonmalfeasance, and justice when deciding whether to perform breast procedures for adolescent patients. ${ }^{56,57}$ Autonomy reflects the view that the patient shares responsibility for decision making about procedures along with the surgeon, provided that they are adequately informed about the risks and benefits of the procedure that they seek. As noted above, adolescents may not be able to fully appreciate the risks and benefits of plastic surgery. Ethically, surgeons are responsible for providing patients and their parents/guardians with accurate information about the desired procedure and for making a determination of the patient's ability to consent to surgery before agreeing to perform surgical procedures on an adolescent. $^{56}$

Beneficence refers to the surgeon's duty to act in the patient's best interests and to maximize benefit to the patient. To date, there is no data supporting the long-term psychosocial benefits of plastic surgery. ${ }^{56}$ However, as reviewed above, there is evidence that adolescents with breast anomalies often experience significant appearance-related distress preoperatively, ${ }^{26}$ and there is some evidence for postoperative psychosocial improvements. ${ }^{17,37}$ Thus, surgeons should consider if the benefits of the procedure outweigh the risks of operating on an adolescent during a period of rapid physical and psychological development. In some instances, delaying surgery until the patient is more physically or emotionally mature may be the most ethical decision even if the patient disagrees with the surgeon's assessment of readiness.

Similarly, nonmalfeasance is the principle that reflects the duty of the surgeon to not cause harm to a patient. For example, operating on adolescent patients with unrealistic expectations or psychiatric disorders such as body dysmorphic disorder may ultimately do more harm than good for the patient. Likewise, performing surgery because a patient demands it even though they have a poor understanding of the long-term impact of surgery may also expose the patient to harm financially, psychologically, and physically.

Finally, the principle of justice or fairness can also factor into the treatment of adolescent patients. Inequities related to access to care are common and often vary as a function of socioeconomic status and insurance coverage. For example, there is variability among insurance companies with respect to coverage for procedures to correct breast asymmetries and gynecomastia. Adolescents whose families have financial resources may be able to pay for these procedures out of 
pocket, whereas others may be unable to afford them or incur debt to pay for them. It is also important to consider whether families understand the long-term financial consequences of having any surgery that involves breast implants, as multiple replacements may be required over the course of the patient's lifetime.

In summary, adolescent breast surgery patients have specific vulnerabilities that necessitate careful consideration of each individual patient's physical, psychological, and cognitive maturity. Surgeons who agree to treat adolescents should be mindful of the legal and ethical considerations related to this patient population, not only to maximize benefits for patients, but also to reduce the likelihood of lawsuits and patient dissatisfaction with postoperative outcomes. Plastic surgery should be recommended only for those adolescents who are psychologically stable, able to thoughtfully participate in the surgery decision-making process, and who have realistic expectations and motivations.

\section{Clinical Management and Screening}

Cosmetic and reconstructive breast surgery are elective procedures, thus successful outcomes are inherently tied to optimal patient selection. ${ }^{58}$ The ASPS's "Plastic Surgery for Teenagers Briefing Paper" purports that successful outcomes typically occur when (1) the adolescent initiates the request for surgery, (2) the teenager has realistic goals for surgery, and (3) the adolescent has demonstrated maturity and can tolerate the physical and emotional changes associated with surgery. ${ }^{59}$ Good clinical management of the adolescent breast surgery patient should include assessment of the patient's knowledge of the desired procedure, motivations and expectations, psychological status, and body-image concerns. ${ }^{60}$

\section{Knowledge of the Procedure}

Although there is no specific procedure for informed consent among adolescents seeking plastic surgery, ${ }^{53}$ adolescent patients should be informed about the procedure and its risks in an understandable manner, be given sufficient time to ask questions, and not be subjected to undue pressure to make a decision about a procedure from parents, peers, or providers. Adolescents, as well as their consenting parents/ guardians, should be able to articulate a basic understanding of how the procedure will be performed, what recuperation will entail (e.g., length of hospital stay, time off from school or activities), risks, and rates of complications. ${ }^{51}$ Alternatives to having surgery, including delaying the timing of surgery until physical maturity is complete or not having surgery at all, should also be discussed. For breast procedures, particularly those involving implants, adolescents and their families should be aware of the possibility for postoperative complications (e.g., scarring, capsular contracture) as well as the possible longer-term effects of breast implants, including those related to sensation, pregnancy, lactation, weight change, and breast cancer screening (e.g., mammograms). They should also be informed of the need to replace implants repeatedly over one's lifetime, as well as the future financial considerations for surgeries to replace implants and the potential that insurance may not cover these procedures or procedures related to complications. Adolescents or parents who state "I hadn't thought about that" or "I don't know" in response to questions or information about breast procedures may benefit from additional time, education, and discussion of risks and benefits before proceeding with surgery.

\section{Motivations and Expectations}

Motivations and expectations for breast surgery should be explored carefully with adolescent patients. ${ }^{60}$ Although body-image dissatisfaction may play an important role in an adolescent's decision to undergo breast surgery, other reasons may also serve as motivations to change their appearance. Pressure from parents to improve appearance may play a role, particularly in families in which a parent has had cosmetic procedures. Mass media may also influence adolescents' interest in breast surgery through promotion of unrealistic images of beauty and stories that highlight the benefits of cosmetic surgery. ${ }^{61}$

Peer pressure can directly or indirectly influence an adolescent's decision to have breast surgery. Adolescents may suggest plastic surgery to peers who are experiencing appearance dissatisfaction or appearance-based teasing; in some communities, plastic surgery may be seen as a rite of passage or given as a gift for a milestone birthday or graduation. These instances raise concerns about the adolescent's ability to independently evaluate and understand the longterm risks and benefits of cosmetic procedures. ${ }^{14,60}$

Inquiring about the timing of surgery can also help identify whether the desire for surgery is motivated by internal factors, such a desire to improve body image, or by external factors, including a desire to eliminate teasing or please others. Individuals who pursue surgery for purely external reasons may be at risk for poor psychological outcomes. ${ }^{62}$ Surgeons should remind adolescents that it is impossible to predict or control how others will respond to their altered appearance. Some adolescents may find that few people notice their changed appearance, while others may receive both positive and negative comments about their postoperative appearance.

\section{Psychological Status and Body Image}

It is important that adolescents presenting for breast surgery be screened for any current or past psychological or psychiatric difficulties as the existence of a psychiatric disorder may make it difficult to determine a patient's ability to make an autonomous decision about surgery and could compromise postoperative outcomes. $^{60,63}$

Adolescents should be asked about their past and current psychological functioning and any history of psychological or psychiatric treatment. Adolescents should also be screened for depression, anxiety, and substance abuse. Parents/guardians should be asked about their adolescent's mental health history as well as concerns about their child's behavior or demeanor. For patients already engaged in psychiatric or psychological treatment, surgeons should ask if their treatment provider is aware of their decision to pursue breast surgery. Mental health providers should be contacted to verify that the timing of the surgery is appropriate. ${ }^{60,63}$ 
As reviewed above, adolescents who seek cosmetic and reconstructive breast procedures may be at risk for eating disorders. Patients with a body mass index $<20 \mathrm{~kg} / \mathrm{m}^{2}$ should be asked about recent weight fluctuations, ongoing dieting efforts, binge eating, purging, and other compensatory behaviors. Young women should also be asked about amenorrhea. ${ }^{63}$ Adolescents who present with symptoms of any psychiatric disorders should be referred to a mental health professional for additional evaluation and treatment before surgery is offered. ${ }^{60,63}$

Body image concerns should also be assessed preoperatively. Patients should be asked what aspects of their appearance bother them. Surgeons can initiate a dialogue about body image by asking patients whether their appearance makes them feel self-conscious or isolated, whether they worry a lot about how they look, or if they avoid places or situations because of their appearance difference. Social stigmatization such as teasing should also be evaluated. ${ }^{63}$

Although some degree of body image dissatisfaction is normative among plastic surgery patients, it is possible that some patients presenting for treatment of objectively mild concerns may be suffering from body dysmorphic disorder (BDD). BDD is a psychiatric condition characterized by preoccupation with an imagined or slight defect in appearance that results in significant distress or impairment in functioning. ${ }^{64}$ Because some concern about physical appearance is normative during adolescence, it may be challenging to diagnose BDD during this developmental period. Careful assessment of an adolescent's appearance concerns and their impact on school and social performance is needed to differentiate normal from pathological appearance concerns. ${ }^{65}$ Individuals with BDD rarely benefit from plastic surgery and should be referred for further psychological and/or psychiatric evaluation. $^{64}$

\section{Conclusions}

Each year, thousands of adolescent patients undergo breast surgery to improve their physical functioning and/or appearance. Although research on the psychological impact of these procedures among adolescents is scant, there is evidence that this patient population is at risk for psychological difficulties. Adolescents also have specific developmental vulnerabilities that necessitate careful consideration of each individual's physical, psychological, and cognitive maturity. Because of these concerns, plastic surgeons should carefully assess each adolescent's knowledge of the procedure, motivations and expectations, as well as psychological stability prior to surgery. Collaboration with mental health professionals is recommended to maximize outcomes for adolescent patients and minimize risks to the plastic surgeon.

\section{References}

1 American Society of Plastic Surgeons. 2011 Plastic Surgery Report. Arlington Heights, IL: American Society of Plastic Surgeons; 2012

2 Sarwer DB, Crerand CE. Body image and cosmetic medical treatments. Body Image 2004;1(1):99-111
3 Sarwer DB, Wadden TA, Pertschuk MJ, Whitaker LA. The psychology of cosmetic surgery: a review and reconceptualization. Clin Psychol Rev 1998;18(1):1-22

4 Sarwer DB, Infield AL, Crerand CE. Plastic surgery for children and adolescents. In: Thompson JK, Smolak, eds. Body Image, Eating Disorders, and Obesity in Youth, 2nd ed. Washington, DC: American Psychological Association; 2008:341-366

5 Cash TF. Body image and plastic surgery. In: Sarwer DB, Pruzinsky T, Cash TF, Goldwyn RM, Persing JA, Whitaker LA, eds. Psychological Aspects of Reconstructive and Cosmetic Plastic Surgery: Clinical, Empirical and Ethical Perspectives. Philadelphia, PA: Lippincott, Williams and Wilkins; 2006:37-59

6 Sarwer DB, LaRossa D, Bartlett SP, Low DW, Bucky LP, Whitaker LA. Body image concerns of breast augmentation patients. Plast Reconstr Surg 2003;112(1):83-90

7 Banbury J, Yetman R, Lucas A, Papay F, Graves K, Zins JE. Prospective analysis of the outcome of subpectoral breast augmentation: sensory changes, muscle function, and body image. Plast Reconstr Surg 2004;113(2):701-707, discussion 708-711

8 Sarwer DB, Gibbons LM, Magee L, et al. A prospective, multi-site investigation of patient satisfaction and psychosocial status following cosmetic surgery. Aesthet Surg J 2005;25(3): 263-269

9 Sarwer DB, Wadden TA, Whitaker LA. An investigation of changes in body image following cosmetic surgery. Plast Reconstr Surg 2002;109(1):363-369, discussion 370-371

10 Simis KJ, Verhulst FC, Koot HM. Body image, psychosocial functioning, and personality: how different are adolescents and young adults applying for plastic surgery? J Child Psychol Psychiatry 2001;42(5):669-678

11 Simis KJ, Hovius SE, de Beaufort ID, Verhulst FC, Koot HM. After plastic surgery: adolescent-reported appearance ratings and appearance-related burdens in patient and general population groups. Plast Reconstr Surg 2002;109(1):9-17

12 U.S. Food and Drug Administration. Breast Implants. Available at: http://www.fda.gov/MedicalDevices/ProductsandMedicalProcedures/ImplantsandProsthetics/BreastImplants/default.html. Accessed October 2, 2012

13 American Society of Plastic Surgeons. Policy Statement: Breast Augmentation in Teenagers. Available at: http://www.plasticsurgery.org/Documents/medical-professionals/health-policy/key-issues/Policy-Statement-on-Breast-Augmentation-in-Teenagers. pdf. Accessed October 2, 2012

14 Crerand CE, Infield AL, Sarwer DB. Psychological considerations in cosmetic breast augmentation. Plast Surg Nurs 2007;27(3): 146-154

15 Sarwer DB. The psychological aspects of cosmetic breast augmentation. Plast Reconstr Surg 2007;120(7, Suppl 1):110S-117S

16 Didie ER, Sarwer DB. Factors that influence the decision to undergo cosmetic breast augmentation surgery. J Womens Health (Larchmt) 2003;12(3):241-253

17 Cash TF, Duel LA, Perkins LL. Women's psychosocial outcomes of breast augmentation with silicone gel-filled implants: a 2-year prospective study. Plast Reconstr Surg 2002;109(6):2112-2121, discussion 2122-2123

18 Fryzek JP, Signorello LB, Hakelius L, et al. Local complications and subsequent symptom reporting among women with cosmetic breast implants. Plast Reconstr Surg 2001;107(1):214-221

19 Sarwer DB, Brown GK, Evans DL. Cosmetic breast augmentation and suicide. Am J Psychiatry 2007;164(7):1006-1013

20 Jacobsen PH, Hölmich LR, McLaughlin JK, et al. Mortality and suicide among Danish women with cosmetic breast implants. Arch Intern Med 2004;164(22):2450-2455

21 Greydanus DE, Matytsina L, Gains M. Breast disorders in children and adolescents. Prim Care 2006;33(2):455-502

22 American Society of Plastic Surgeons. Position Paper: Gynecomastia. Arlington Heights, IL: American Society of Plastic Surgeons; 2002 
23 Fisher M, Fornari V. Gynecomastia as a precipitant of eating disorders in adolescent males. Int J Eat Disord 1990;9(1):115-119

24 Ridha H, Colville RJI, Vesely MJJ. How happy are patients with their gynaecomastia reduction surgery? J Plast Reconstr Aesthet Surg 2009;62(11):1473-1478

25 Storch EA, Lewin AB, Geffken GR, et al. Psychosocial adjustment of two boys with gynecomastia. J Paediatr Child Health 2004;40(56):331

26 Kinsella C Jr, Landfair A, Rottgers SA, et al. The psychological burden of idiopathic adolescent gynecomastia. Plast Reconstr Surg 2012;129(1):1-7

27 American Society of Plastic Surgeons. Report of the 2010 Plastic Surgery Statistics. Arlington Heights, IL: American Society of Plastic Surgeons; 2011

28 Young VL, Watson ME. Breast reduction. In: Sarwer D, Pruzinsky T, Cash T, Goldwyn R, Persing J, Whitaker L, eds. Psychological Aspects of Cosmetic and Reconstructive Surgery. Philadelphia, PA: Lippincott, Williams \& Wilkins; 2006:189-206

29 Koltz PF, Sbitany H, Myers RP, Shaw RB, Patel N, Girotto JA Reduction mammaplasty in the adolescent female: the URMC experience. Int J Surg 2011;9(3):229-232

30 Saariniemi KM, Joukamaa M, Raitasalo R, Kuokkanen HO. Breast reduction alleviates depression and anxiety and restores selfesteem: a prospective randomised clinical trial. Scand J Plast Reconstr Surg Hand Surg 2009;43(6):320-324

31 ACOG Committee on Adolescent Health Care. ACOG Committee Opinion No. 350, November 2006: Breast concerns in the adolescent. Obstet Gynecol 2006;108(5):1329-1336

32 Cerrato F, Webb ML, Rosen H, et al. The impact of macromastia on adolescents: a cross-sectional study. Pediatrics 2012;130(2): e339-e346

33 Brown AP, Hill C, Khan K. Outcome of reduction mammaplasty-a patients' perspective. Br J Plast Surg 2000;53(7):584-587

34 Dabbah A, Lehman JA Jr, Parker MG, Tantri D, Wagner DS. Reduction mammaplasty: an outcome analysis. Ann Plast Surg 1995;35 (4):337-341

35 Behmand RA, Tang DH, Smith DJJ Jr. Outcomes in breast reduction surgery. Ann Plast Surg 2000;45(6):575-580

36 Shakespeare V, Cole RP. Measuring patient-based outcomes in a plastic surgery service: breast reduction surgical patients. Br J Plast Surg 1997;50(4):242-248

37 Rogliani M, Gentile P, Labardi L, Donfrancesco A, Cervelli V. Improvement of physical and psychological symptoms after breast reduction. J Plast Reconstr Aesthet Surg 2009;62(12):1647-1649

38 Cruz-Korchin N, Korchin L, González-Keelan C, Climent C, Morales I. Macromastia: how much of it is fat? Plast Reconstr Surg 2002;109(1):64-68

39 Wagner DS, Alfonso DR. The influence of obesity and volume of resection on success in reduction mammaplasty: an outcomes study. Plast Reconstr Surg 2005;115(4):1034-1038

40 Collins ED, Kerrigan CL, Kim M, et al. The effectiveness of surgical and nonsurgical interventions in relieving the symptoms of macromastia. Plast Reconstr Surg 2002;109(5):1556-1566

41 Evans GR, Ryan JJ. Reduction mammaplasty for the teenage patient: a critical analysis. Aesthetic Plast Surg 1994;18(3): 291-297

42 Lee MC, Lehman JA Jr, Tantri MDP, Parker MG, Wagner DS. Bilateral reduction mammoplasty in an adolescent population: adolescent bilateral reduction mammoplasty. J Craniofac Surg 2003;14 (5):691-695

43 Webb ML, Cerrato F, Rosen H, DiVasta AD, Greene AK, Labow BI. The effect of obesity on early outcomes in adolescents undergoing reduction mammaplasty. Ann Plast Surg 2012;68(3):257-260

44 McMahan JD, Wolfe JA, Cromer BA, Ruberg RL. Lasting success in teenage reduction mammaplasty. Ann Plast Surg 1995;35(3): 227-231
45 Losee JE, Serletti JM, Kreipe RE, Caldwell EH. Reduction mammaplasty in patients with bulimia nervosa. Ann Plast Surg 1997;39 (5):443-446

46 Kreipe RE, Lewand AG, Dukarm CP, Caldwell EH. Outcome for patients with bulimia and breast hypertrophy after reduction mammaplasty. Arch Pediatr Adolesc Med 1997;151(2):176-180

47 Losee JE, Jiang S, Long DE, Kreipe RE, Caldwell EH, Serletti JM. Macromastia as an etiologic factor in bulimia nervosa: 10-year follow up after treatment with reduction mammaplasty. Ann Plast Surg 2004;52(5):452-457, discussion 457

48 van Aalst JA, Sadove AM. Treatment of pediatric breast problems. Clin Plast Surg 2005;32(1):65-78, ix

49 Lin KY, Nguyen DB, Williams RM. Complete breast absence revisited. Plast Reconstr Surg 2000;106(1):98-101

50 Oakes MB, Quint EH, Smith YR, Cederna PS. Early, staged reconstruction in young women with severe breast asymmetry. J Pediatr Adolesc Gynecol 2009;22(4):223-228

51 McGrath MH, Schooler WG. Elective plastic surgical procedures in adolescence. Adolesc Med Clin 2004;15(3):487-502

52 Piza-Katzer H. Reduction mammaplasty in teenagers. Aesthetic Plast Surg 2005;29(5):385-390

53 Zuckerman D, Abraham A. Teenagers and cosmetic surgery: focus on breast augmentation and liposuction. J Adolesc Health 2008;43 (4):318-324

54 Steinberg L. Risk taking in adolescence: new perspectives from brain and behavioral science. Curr Dir Psychol Sci 2007;16(2):5559

55 Kessler RC, Amminger GP, Aguilar-Gaxiola S, Alonso J, Lee S, Ustün TB. Age of onset of mental disorders: a review of recent literature. Curr Opin Psychiatry 2007;20(4):359-364

56 Laneader A, Wolpe PR. Ethical considerations in cosmetic surgery. In: Sarwer DB, Pruzinsky T, Cash TF, Goldwyn RM, Persing JA, Whitaker LA, eds. Psychological Aspects of Reconstructive and Cosmetic Plastic Surgery: Clinical, Empirical, and Ethical Perspectives. Philadelphia, PA: Lippincott, Williams, \& Wilkins; 2006: 301-314

57 Sterodimas A, Radwanski HN, Pitanguy I. Ethical issues in plastic and reconstructive surgery. Aesthetic Plast Surg 2011;35(2): 262-267

58 DeSilva NK. Plastic surgery and the adolescent breast: preliminary patient counseling. J Pediatr Adolesc Gynecol 2010;23(3): 184-186

59 American Society of Plastic Surgeons. Plastic Surgery for Teenagers Briefing Paper. Available at: http://www.plasticsurgery.org/ News-and-Resources/Briefing-Papers/Plastic-Surgery-for-Teenagers.html. Accessed October 2, 2012

60 Sarwer DB. Psychological assessment of cosmetic surgery patients. In: Sarwer DB, Pruzinsky T, Cash TF, Goldwyn RM, Whitaker LA, eds. Psychological Aspects of Reconstructive and Cosmetic Plastic Surgery: Clinical, Empirical, and Ethical Perspectives. Philadelphia: Lippincott, Williams \& Wilkins; 2006:267-286

61 Pearl A, Weston J. Attitudes of adolescents about cosmetic surgery. Ann Plast Surg 2003;50(6):628-630

62 Honigman RJ, Phillips KA, Castle DJ. A review of psychosocial outcomes for patients seeking cosmetic surgery. Plast Reconstr Surg 2004;113(4):1229-1237

63 Crerand CE, Magee L. Reconstructive procedures. In: Block AR, Sarwer DB, eds. Presurgical Psychological Screening: Understanding Patients, Improving Outcomes. Washington, DC: American Psychological Association; 2012:173-194

64 Crerand CE, Franklin ME, Sarwer DB. Body dysmorphic disorder and cosmetic surgery. Plast Reconstr Surg 2006;118(7): 167e-180e

65 Hadley SJ, Greenberg J, Hollander E. Diagnosis and treatment of body dysmorphic disorder in adolescents. Curr Psychiatry Rep 2002;4(2):108-113 